\title{
Blended learning para un programa de desarrollo de habilidades informacionales en la Biblioteca de la Escuela Universitaria de Formación de Profesorado de Lugo
}

Blended learning for a programme on the development of information abilities in the library of the Education School of Lugo

\author{
Carmen Varela Prado \\ Universidad de Santiago de Compostela (España)
}

\begin{abstract}
Resumen
En la biblioteca de la Escuela de Formación de Profesorado de Lugo se ha desarrollado en el curso 2006-2007 un programa de Alfabetización Informacional con un sistema combinado de estrategias de aprendizaje, presencial y virtual de forma simultánea y complementaria (blended-learning).
\end{abstract}

Palabras clave: Alfabetización informacional. Desarrollo de habilidades informativas. Formación de usuarios. Espacios virtuales de aprendizaje. Innovación educativa.

\section{Introducción}

Es mucho lo que se lleva escrito en estos últimos años sobre conceptos como: innovación, enseñanza aprendizaje, construcción de conocimiento, aprendizaje continuo... Son conceptos que entendemos perfectamente; y también, cuales son los principios que deberían desarrollarse en cada uno de ellos para adaptarnos a las exigencias de la nueva sociedad del conocimiento. Sin embargo, observamos que pocas medidas prácticas se han adoptado para afrontar esa realidad. Las universidades tienen una gran parte de responsabilidad en conducir a la sociedad hacia el cambio con nuevas políticas y modelos de docencia, de investigación para la adquisición de competencias transferibles. El modelo de enseñanza/aprendizaje, de universidad investigadora, emprendedora e innovadora, los distintos tipos de competencias, el aprendizaje a lo largo de la vida, son postulados que podemos ver plasmados en todos los códigos legislativos - Ley Orgánica de Universidades (LOU), estatutos...- pero más bien parecen quedarse en una declaración de intenciones. Innovar no significa renovar, debe de significar

\begin{abstract}
The library of La Escuela Universitaria de Formación del Profesorado of Lugo has elaborated an Information Literacy programme in 2006-2007, which consists on the simultaneus combination of several classes in wich atudents have to be present and others that are conducted virtually (blended-learning)
\end{abstract}

Key Words: Information literacy. Development of information habilities. Users education. Virtual learning platforms.

un cambio deliberadamente asumido en todos los colectivos que conformamos la comunidad educativa que incluya la flexibilidad mental, la adopción del cambio y el aprendizaje continuo. La investigación sobre la enseñanza esta adelantada de acuerdo a la Convergencia Europea, pero no así la acción que debería estar implementada en la realidad de las aulas, en la mentalidad del docente magistral y en la innovación de los recursos y metodologías utilizadas.

También esta problemática afecta a los bibliotecarios/as y documentalistas universitarios como colectivo responsable de la gestión de los recursos de información y del asesoramiento de profesores y alumnos en su uso y que en este nuevo marco nos va a tocar jugar un papel trascendental, porque el acceso a la información cada vez se complica mas y los usuarios cada vez necesitan mas ayuda para aprender a buscar, recuperar y seleccionar la información adecuada a su necesidad informativa.

Los bibliotecarios/as universitarios tenemos unas funciones muy específicas y todavía muy ligadas a la conservación, a procesos, a la difusión bajo demanda y a la formación unidireccio- 
nal que representamos en nuestras sesiones de formación de usuarios. El perfil del nuevo bibliotecario/a y los métodos utilizados en la formación de usuarios, responden en poca medida a las demandas del nuevo modelo de enseñanza/aprendizaje. Considero que para afrontar procesos de formación en alfabetización, además de tener los conocimientos que se nos suponen en información y documentación, es necesario tener una formación pedagógica, conocer nuevas metodologías y recursos de enseñanza más atractivos y novedosos, además de las que se requieran en tecnología de la información y comunicación. Es difícil asumir esta polivalencia necesaria para abordar proyectos de formación con éxito que además habrá que sumarla a la que se precisa para abordar el resto de las funciones y tareas diarias que también están sujetas a cambio constantes y que hace preciso un reciclaje continuo.

En este trabajo pretendo exponer una experiencia de formación en Alfabetización Informacional llevada a las aulas y a su vez continuada en un espacio distinto vivo y abierto, una experiencia nueva en la que sumamos nuevas metodologías de aprendizaje, tecnología, recursos de información y nuevas técnicas de trabajo.

En primer lugar procede establecer el marco de actuación, el estado de la cuestión en que se desarrolla esta experiencia concreta, aspectos sociales, educativos y metodológicos para dar paso a la planificación, desarrollo y análisis de resultados del Programa de Desarrollo de Habilidades Informacionales (DHI) en la biblioteca de la Escuela Universitaria de Formación de Profesorado de Lugo, Universidad de Santiago de Compostela.

\section{Estado de la cuestión}

La nueva sociedad del conocimiento es considerada fuente de riqueza y de valor en la actual economía mundial. En el mercado laboral se ha elevado en un grado muy importante la competitividad entre los trabajadores en cuanto al nivel de sus conocimientos y al valor añadido que sean capaces de generar.

Ante esta situación, la universidad no puede ser simplemente espectadora frente a la necesidad social que ya es real y palpable, y mucho menos si tenemos en cuenta las directrices marcadas por el espacio Europeo de Educación Superior, en las que claramente se potencia la idea de un aprendizaje continuo. Dentro de esta línea se debería optar por un paradigma pedagógico basado en la educación a lo largo de la vida que tenga como fundamento el hecho de que la sociedad está sujeta a un proceso permanente de aprendizaje, pues las situaciones cambian, evolucionan y se crean nuevos perfiles y necesidades.

A este nuevo esquema conocimiento/ aprendizaje tiene que responder la universidad con cambios en políticas, estilos y metodologías de enseñanza determinando nuevos patrones donde los principios básicos sean: enseñar a aprender, la formación continua sin tener en cuenta el espacio y el tiempo. Maria Pinto (2006) dice:

que el principal reto de los sistemas educativos se-
rá potenciar la adquisición, estructuración y organi-
zación del conocimiento, desarrollando el capital
intelectual de los estudiantes y dotándolos de un
amplio inventario de habilidades y competencias
para la resolución de problemas. Es necesario po-
tenciarles la capacidad de aprender a aprender, de
promover su crecimiento integral, de estimular el
desarrollo de la inteligencia para sistematizar y re-
lacionar conocimientos, para innovar y crear futuro.
El aprendizaje ha de ser un proceso continuo,
flexible e innovador, que fomente a lo largo de toda
la vida el desarrollo de habilidades y capacidades
en un mundo en constante cambio.

La tecnología favorece este nuevo modelo de enseñanza que centra su atención en facilitar la capacidad de construir conocimiento, puesto que el conocimiento no se puede transmitir listo para consumir sino que debe construirse en las mentes de los alumnos. En este sentido, las tecnologías de la información y comunicación además de favorecer la vuelta a la esencia del aprendizaje continuo planteado ya por los precursores de la pedagogía en el siglo XIX y, sobre todo, en las teorías constructivitas de Piaget- modifican los procesos de comunicación al transformar el modo de producir, procesar, almacenar, transferir y acceder a la información, creando así nuevas posibilidades de interaccionar y de relacionarse con la misma.

También la nueva biblioteca universitaria, biblioteca híbrida, flexible y sin muros tiene la responsabilidad de involucrarse activamente y participar en el desenvolvimiento del modelo de aprendizaje significativo y constructivo, porque tiene la capacidad del control de la información, porque siempre fue pionera en asumir los avances de la tecnología y por su potencial en recursos humanos especializado y profesional para enfrentarse a los nuevos retos de la Convergencia Europea y a las innovaciones tecnológicas para construir un capital intelectual que pueda liderar la innovación en la enseñanza/aprendizaje. 


\section{Formación de Usuarios v. Alfabetización Informacional}

La introducción del concepto de alfabetización informacional en el ámbito de la biblioteca universitaria marca un profundo cambio en la pedagogía de la formación de usuarios, que de estar centrada en el bibliotecario que imparte formación a usuarios individuales o en grupo pasará a estar centrada en el estudiante y en el aprendizaje independiente como demuestra el uso de una variedad de técnicas de formación que tratan de satisfacer las necesidades de usuarios diversos.

Este cambio afecta a las formas de impartir la formación, evolucionando desde las visitas guiadas a la biblioteca y las sesiones de formación, a unidades integradas de servicio que cubren las prácticas de búsquedas de información, caracterizadas por la provisión de recursos de autoayuda de carácter general y actividades sobre temas específicos.

Según algunos autores, el problema de la formación de usuarios tradicional es que se centra en aspectos específicos de la actividad bibliotecaria que cubren el uso de herramientas de información, pero no exploran tareas más complejas de la recuperación de la información, como el pensamiento crítico y la evaluación.

Lichtenstein, se queja de que la practica habitual en la formación de usuarios esta enfocada a actividades de presentación de la biblioteca, sus servicios, la disposición de los fondos, pero no se enseña a los estudiantes como llegar a estar alfabetizados en información.

De todas formas, la formación de usuarios es una disciplina madura en la biblioteconomía universitaria, pero su definición y alcance se cuestiona hoy en día. La alfabetización en información ve la formación de usuarios en un marco más amplio por lo que se ha convertido en el nuevo lenguaje de este campo (Aragón González, 2005, p. 429). En la actualidad, se entiende por alfabetización informacional, el desarrollo de habilidades destrezas y actitudes para gestionar la información con eficacia. La Alfabetización Informacional en la universidad tiene la finalidad de formar nuevas generaciones de estudiantes competentes y entrenados para explorar en el mundo de la información, saber buscar, evaluar y seleccionar la información más pertinente a su necesidad, saber integrarla en su conocimiento previo, construir un nuevo conocimiento y saber usarlo adecuadamente (Pinto Molina, 2006):

Como señala la UNESCO, los estudiantes universitarios deben ser ciudadanos bien informados y mo- tivados, en permanente autoformación y capacitados para analizar los problemas sociales, buscar soluciones, aplicarlas y asumir responsabilidades.

Según Maria Pinto, algunos de los factores que influyen en la alfabetización informacional son:

- Crecimiento exponencial de la información disponible y accesible en cualquier formato

- Necesidad de que cada persona adquiera y desarrolle aptitudes transferibles y utilizables a lo largo de toda la vida para la resolución de problemas.

- La evolución hacia una pedagogía activa centrada en el estudiante, basada en los recursos y en la solución de problemas en contexto

El estudiante que quiera dominar el acceso y uso de la información tanto para su aprendizaje como para el desempeño profesional, deberá poseer o adquirir un conjunto de competencias y habilidades específicas. De forma clara y precisa, estas habilidades y destrezas son expuestas por María Pinto Molina en e-coms, portal que ella dirige.

Estas recomendaciones tienen como antecedente las Normas sobre Alfabetización en Enseñanza Superior (ACRL/ALA 2000), aprobadas y adoptadas por la American Association for Higher Education y los organismos de acreditación estadounidenses. Formulan estándares para el dominio de competencias básicas y sugieren indicadores y resultados observables de su consecución.

Según la norma, una persona competente en el acceso y uso de la información, es aquella que es capaz de reconocer sus necesidades informativas, que sabe localizar la información, procesarla, evaluarla y utilizarla.

Estas normas estadounidenses han sido revisadas y adoptadas por el Council of Australian University Libraries (CAUL 2001), como Normas sobre alfabetización en información para las bibliotecas universitarias australianas y neozelandesas. Proponen siete normas para adiestrar al estudiante y medir su aprendizaje en alfabetización de información.

De la misma forma en Gran Bretaña, a través de la Society of College, National and University Libraries (SCONUL) se han tratado también las competencias en alfabetización que debían tener los estudiantes. En 1999 se elabora un documento en el que se habla de 7 pilares básicos que son necesarios para la gestión y uso de la información en la misma línea del el ALA y CAUL. La propuesta de SCONUL constituye el documento base sobre el que se fundamenta la competencia en alfabetización informativa obje- 
to de estudio, del cual relaciono los siete puntos que significan el punto de arranque dentro de los contenidos de la experiencia de formación.

1. Reconocer la necesidad de información.

2. Distinguir entre las diferentes formas de tratamiento de la necesidad de información reconocida.

3. Formular estrategias de búsquedas.

4. Localizar y acceder a la información.

5. Comparar y evaluar la información obtenida en diferentes fuentes.

6. Organizar, aplicar y comunicar la información de forma adecuada.

7. Sintetizar y edificar a partir de la información existente, contribuyendo a la creación de un nuevo conocimiento.

\section{Metodología y Herramientas}

Tenemos que tener en cuanta que en la Alfabetización informacional entran en juego nuevos escenarios además del físico y presencial que son los que posibilitan las tecnologías de la información y comunicación. Adaptarse a ellos significa también un cambio y otro reto por su versatilidad, lo cual supone adquirir nuevas habilidades y destrezas que nos permitan adaptarnos. El dominio de las tecnologías de la información se considera competencia básica en los planes de estudio desde los primeros años y su mención está presente en todos y cada uno de los distintos códigos legislativos educativos.

La implantación de las TIC ha provocado el cambio más importante en la metodología utilizada en Alfabetización Informacional, incrementándose el aprendizaje activo gracias a que la tecnología permite hacer presentaciones multimedia con gran cantidad de actividades prácticas para los estudiantes. Internet ha cambiado drásticamente la formación y el aprendizaje desde el momento que permite el acceso a la información y la comunicación atravesando fronteras, culturas a escala global. Ofrece el potencial para compartir recursos de aprendizaje, compartir información y trabajar en grupo (ibidem, 2006); estamos hablando de los espacios virtuales de aprendizaje o teleformación

Hay que considerar las ventajas que presenta el e-learning como herramienta para el aprendizaje.

- Gran flexibilidad en el acceso a la formación: el estudiante decide el lugar y la hora de estudio.

- Los recursos de e-learning se pueden desarrollar fácilmente utilizando una gran variedad de paquetes estándar
- Es un tipo de formación relativamente barata: No tiene costes de impresión ni de distribución.

- Permite una fácil comunicación entre estudiantes, profesores y bibliotecarios en tiempo real y asíncrono.

- Permite la comunicación uno a uno y uno a muchos.

Si en la formación integramos la modalidad presencial tradicional y el e-learning, obtenemos una modalidad nueva, mixta a la que llamamos complex o blended learning, que concretamente es la utilizada en este programa de formación.

El Blended learning es un aprendizaje en escenarios múltiples donde se combinan actividades presenciales, síncronas y de e-learning como una modalidad integrada de aprendizaje. Es decir, la enseñanza formativa a distancia se completa con sesiones presenciales en el aula o al revés. Lo que se consigue es que el estudiante experimenta varias formas de relación.

En resumen, la formación de usuarios -concepto sustituido por otro de mayor alcance como es la alfabetización informativa-, ha de tener en cuenta además de los siete pilares en los que se basa para considerar a un individuo alfabetizado, en que un estudiante ha de:

- "Aprender haciendo": buscando información, conociendo las fuentes, porque el alumno aprende mientras busca, trabaja, investiga...

- "Cuando quiera o siempre": de forma presencial o a distancia, porque cada vez nos encontramos con un abanico mas amplio de tipos de estudiantes que demandan alternativas a la formación presencial y la tecnología hoy permite asegurar el aprendizaje continuo, autónomo y colaborativo.

- "Con tecnología": porque cada vez la infraestructura tecnológica llega a mas gente, porque la universidad cada vez pone mas medios, porque cada vez es mas fácil trabajar en red y porque los jóvenes acceden a la universidad con un bagaje tecnológico cada vez mas especializado.

- "Con recursos y ayuda": porque la biblioteca tiene la capacidad de poner en línea todo tipo de recursos: impresos, digitales propios, electrónicos propios o externos, guías, tutoriales, materiales de ayuda, etc., y de ofrecer ayuda personalizada o grupal con la utilización de herramientas síncronas y asíncronas.

- "Para Aprender a Aprender": poder adquirir competencias transferibles, habilidades, destrezas y actitudes para gestionar la informa- 
ción con eficacia... ser aprendientes a lo largo de la vida, tomar decisiones y resolver problemas.

\section{Programa de Desarrollo de Habilidades Informacionales}

\subsection{El Programa}

Este programa responde a una iniciativa personal, el modelo es original y adaptado a las características de un colectivo de alumnos en concreto. En su elaboración, he tenido presentes los conocimientos sobre alfabetización Informacional adquiridos a través de la producción de Maria Pinto, la experiencia vivida y relatada por Jose Zapirain Sagaseta en la Davis Library of the University of North Carolina at Capel Hill, la investigación sobre biblioteca electrónica y espacios integrados de información de Ricardo Acebes Jiménez y la propia experiencia adquirida en la realización del master en e-Learning: TIC para la educación y la formación de la Universidad de Santiago de Compostela en el curso 2003-2004.

La acción concreta que aquí voy a exponer responde, por una parte al nivel avanzado del Programa de Alfabetización Informacional incluido en el Programa de Competencias Básicas que se desarrolla en la Escuela de Formación del Profesorado de Lugo desde hace dos años.

Por otra parte, responde a una acción de formación de tipo curricular desarrollada en colaboración con la profesora titular de la materia Bases Psicológicas en Educación

\subsection{Participantes}

Los alumnos de tercer curso de la diplomatura de Magisterio de las cuatro especialidades: Infantil, Primaria, Educación física y Lenguas extranjeras que suman en total 146 alumnos. La profesora colaboradora que ejerce la función de asesora y tutora de contenidos y yo la tutorización metodológica y documental.

Tenemos que tener en cuenta también los siguientes factores que van a influir en la modalidad de formación seleccionada:

1. Cada vez es mas variado el tipo de alumnos que acuden a las aulas universitarias. Cada vez es mayor la convivencia en la universidad de jóvenes y mayores de 25 años que acceden a completar estudios, que deciden volver a estudiar, que trabajan, tienen familia y la vida mas complicada. Esto hace que no puedan asistir a clase con regularidad, y que muchos tengan la dispensada la asistencia a clases.

2. Los alumnos de tercer curso pasan casi dos meses en el primer cuatrimestre haciendo prácticas en las escuelas a las que se les destina y no siempre en la propia ciudad.

3. La implantación de los créditos ECTS requiere que los estudiantes realicen en las distintas materias una parte del trabajo de forma autónoma, con el fin de que desarrollen su capacidad investigadora, y el profesor actúa como asesor, como guía y apoyo.

La implementación de un modelo de enseñanza a distancia a través de una plataforma de teleformación significa una alternativa a la presencialidad y contribuye a favorecer la comunicación, la inteconexión entre los participantes, el trabajo autónomo y colaborativo.

\subsection{Planificación}

Como ya expuse anteriormente, esta acción formativa no es un hecho aislado sino que forma parte de un programa que tiene establecida su misión, sus metas, objetivos, planes de acción y control. La misión del programa es que los alumnos de la Escuela de Formación del Profesorado de Lugo puedan adquirir competencias informacionales.

La meta correspondiente al nivel avanzado del programa es que los alumnos llegan a desarrollar habilidades para plantear su necesidad informativa, buscar y seleccionar información pertinente utilizando las fuentes apropiadas y analizar, contrastar, sintetizar y llegar a construir un nuevo conocimiento.

Para alcanzar esta meta, se establecen una serie de objetivos concretos y los consiguientes planes de actuación.

1. Saber afrontar y plantear racional y ordenadamente la necesidad informativa.

2. Aprender a extraer conceptos y traducirlos en palabras clave.

3. Aprender a elaborar estrategias de búsqueda.

4. Saber seleccionar las fuentes de información adecuadas al tema que traten.

5. Aprender a analizar y seleccionar la información pertinente y contrastarla.

6. Aprender a construir su propio conocimiento, teniendo en cuenta los documentos utilizados dando cuenta de su autoría y haciendo referencia a ellas con una citación correcta. 
7. Elaboración de la bibliografía según estándares normalizados.

El programa se desarrolla fundamentalmente en el primer cuatrimestre del curso utilizando un modelo complex o denominado también Blended learning, combinando la enseñanza en el aula utilizando las horas de practicas adjudicadas a la materia y el e-learning que complementa el aprendizaje presencial y lo hace continuo.

\subsection{El Aula virtual de Aprendizaje}

Este espacio se abre como alternativa y como complemento de la formación que comienza en el aula, y busca transferir lo más fielmente posible los contenidos, la metodología, los materiales y la formación compartida estableciéndose un ambiente de aula abierto y sin horario.

Esta aula se abre en una plataforma que actúa como herramienta para facilitar el aprendizaje y la información en red. Se abre con la denominación de "Curso de D.H.I.: Uso y gestión de la información para los alumnos de la escuela Universitaria de Formación del Profesorado de Lugo". Funciona como curso en el plazo de duración del desarrollo de esta experiencia. Posteriormente el aula sigue abierta a los alumnos inscritos y que necesiten o quieran utilizarla.

La Plataforma seleccionada es de software libre -Doleos-, adaptada por el Centro de Alta Computación de Galicia (CESGA), que la pone a disposición de toda la comunidad educativa e investigadora de Galicia.

En este edificio virtual organizamos los distintos espacios con sus funciones propias. Los destinados a albergar los materiales de aprendizaje y los destinados a la comunicación (figura 1).

1. Espacios de comunicación:

- Espacio de información sobre el aula y la descripción del curso.

- Agenda y calendario de actividades.

- Tablón de anuncios para lanzar comunicados a usuarios determinados o a todos los registrados.

- Foros especializados.

- Chat para tutorías.

- Usuarios y grupos de usuarios registrados

2. Espacios de materiales:

- Documentos: materiales para el aprendizaje, tutoriales, biblioteca digital.

- Enlaces: biblioteca electrónica.
- Trabajos: espacio de recepción de actividades, ejercicios y trabajo final.

- Glosario de la materia.

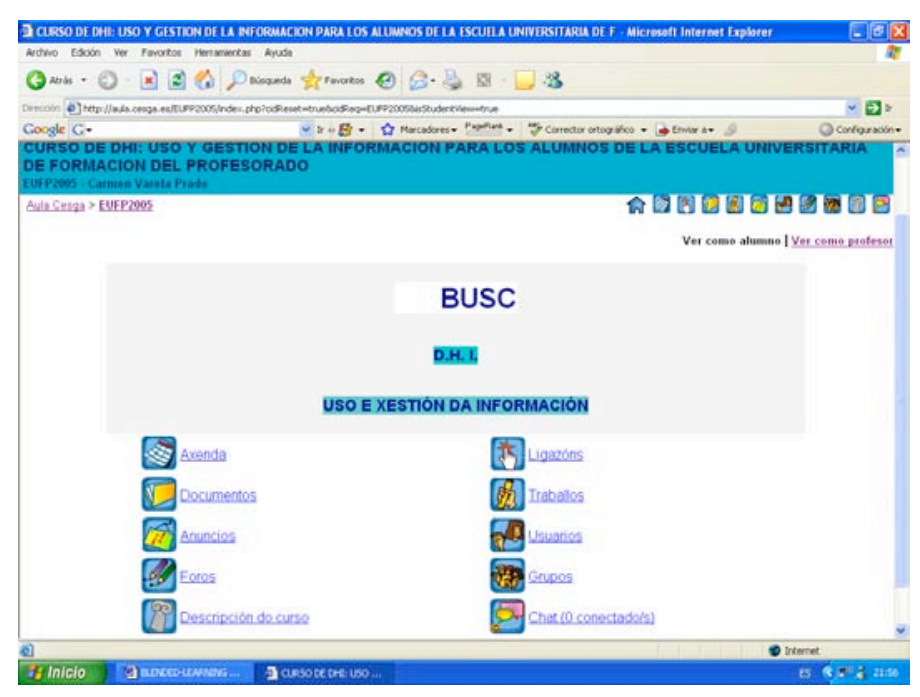

Fig.1. Pantalla de Inicio del Aula virtual

\subsection{Pasos para el desarrollo del Programa}

A lo largo del desarrollo de este curso se va produciendo paulatinamente una intercomunicación entre los alumnos que participan, la profesora, la bibliotecaria y los recursos para ir alcanzando los objetivos propuestos por la profesora responsable de la materia en cuanto a conocimiento y los objetivos de este programa en cuanto a metodología de investigación teniendo en cuenta los siete pilares básicos en los que se fundamenta la Alfabetización Informacional.

Los pasos que se siguen son los siguientes:

1. Del programa de la materia, la profesora selecciona siete bloques o grandes temas y los oferta en clase. Se forma grupos de diez alumnos y seleccionan un tema cada grupo.

2. En la primera clase práctica se presenta la Plataforma, su funcionamiento, los materiales, las herramientas de comunicación, la biblioteca electrónica y como inscribirse en el curso. Al mismo tiempo, se abren los foros con la denominación de cada uno de los temas propuestos. A través de ellos, los alumnos podrán intercambiar información y la profesora intervenir, moderar y orientar.

3. Los grupos formados se subdividen en grupos de tres personas que trabajarán sobre un aspecto concreto de cada tema. Esta tarea la consensúan entre ellos, asesorados 
por la profesora. Esta actividad se desarrolla en clase y a través de los foros.

4. En la clases prácticas sucesivas y una vez que cada grupo tiene decidido su tema - por lo que ya se les supone un conocimiento previo sobre la materia y cuentan con las orientaciones y pautas dadas por la profesora que figuran colgadas en la plataforma-, se comienza a trabajar en la extracción de conceptos, la trascripción en palabras-clave, la búsqueda de términos alternativos y sinónimos. A través de la carpeta de trabajos cada grupo sube sus conclusiones que son valoradas por la profesora y discutidas en clase y a través de los foros. Todas las actividades están previamente anunciadas en el Aula virtual, todos los materiales colgados, los foros abiertos y unas horas determinadas de Chat para solucionar dudas o encaminar a quien se halla rezagado. Esto permite que aunque un alumno/a no asista a clase pueda desarrollar su trabajo, colaborar con su grupo en la distancia y en cualquier tiempo.

5. Presentamos las fuentes de información mas relacionadas con la materia y a las que pueden enlazar desde la Biblioteca Electrónica creada en la Plataforma. Los tutoriales correspondientes los pueden encontrar en la carpeta Documentos/Tutoriales, aunque se les recomienda que consulten las bases de datos en red que oferta la BUSC con el fin de que se acostumbren a navegar por la Web de la Biblioteca Universitaria de Santiago de Compostela.

6. Comenzamos a trabajar en estrategias de búsqueda, búsquedas combinadas, uso de operadores. Los alumnos, una vez que han seleccionados las fuentes de información adecuadas $-y$ que, además, ya fueron comentadas a través de los foros o el Chat-, empiezan a lanzar sus primeras búsquedas expertas y a ver los primeros resultados. Esta es la parte que da más trabajo, tienen que jugar con los términos seleccionados, sustituirlos por otros, traducirlos al inglés, las búsquedas les hacen demasiado ruido o les da cero resultados y son más frecuentes las intervenciones y la solicitud de ayuda.

7. Una vez que cada grupo ha localizado un número de documentos aceptable -libros, artículos de revistas, recursos electrónicos en Internet-, se les da las pautas necesarias para que evalúen la información obtenida con criterios de calidad.

8. Por su parte, empieza el trabajo de elaboración del tema, para ello cuentan con las pautas dadas por la profesora, recomendaciones elaboradas para el análisis, la reflexión, la síntesis y presentación del nuevo conocimiento, así como las normas para la elaboración de un resumen documental y una bibliografía. A partir de este momento, es frecuente que envíen borradores para la corrección, que hagan múltiples y variadas consultas. En definitiva, los foros se hacen muy activos comunicándose entre ellos; y, de vez en cuando, intervenimos las profesoras cuando vemos que algún tema de discusión lleva una orientación descaminada.

9. Por último y en un plazo establecido ya desde el inicio del curso, los alumnos enlazan cada parte de cada bloque o tema general y los suben a la plataforma. La profesora hace las correcciones pertinentes en cuanto a los contenidos, y quedan a disposición de todos para la preparación del examen; suben a la biblioteca digital los documentos electrónicos utilizados, las referencias procedentes de bases de datos y los registros bibliográficos de los catálogos que se organizan por temas. Además, a lo largo del curso han ido construyendo un glosario específico sobre la materia que tienen también a su disposición.

10.Para la evaluación desde la biblioteca, cada subgrupo presentará un informe con todos los pasos dados: el tema, aspecto, conceptos, descriptores, estrategias, fuentes consultadas, un resumen documental y la bibliografía según norma ISO y UNE.

\subsection{Evaluación}

Se utilizan varios criterios de evaluación:

- Evaluación del aula virtual a través de los resultados estadísticos de uso dados por el propio sistema: número de accesos al aula, documentos, enlaces desde la biblioteca electrónica, participación en los foros, consultas en el chat, llegando a obtener una valoración cuantitativa de accesos por alumno (tabla 1).

\begin{tabular}{ll}
\hline Accesos & 2778 \\
\hline Documentos & 2775 descargas \\
\hline Foros & 209 sesiones \\
\hline Actividades y ejercicios & 1105 \\
\hline
\end{tabular}

Tabla I. Actividad en el aula

- Evaluación del Programa a través de una Encuesta de satisfacción. De las 145 respuestas recibidas, el grado de satisfacción se recoge en la tabla 2 . 


\begin{tabular}{ccc}
\hline Poco satisfecho & Satisfecho & Muy satisfecho \\
\hline 3 & 42 & 101 \\
\hline
\end{tabular}

Tabla II. Grado de satisfacción

Tenemos que destacar que una vez finalizado el curso tanto de forma personal como por correo electrónico muchos de los estudiantes preguntaban y demandaban el poder seguir teniendo acceso al aula, a los materiales y a poder establecer comunicación y obtener ayuda para otras materias.

Por fin, la evaluación cualitativa de los alumnos se realiza en función de los siguientes criterios:

- Participación en el aula y los foros.

- Valoración de las distintas actividades realizadas.

- Valoración cualitativa de los temas elaborados.

- Calificación obtenida en el examen con respecto a esta parte.

- Evaluación del informe presentado.

\section{Resultados}

En cuanto a la participación en el aula y en los foros resultó muy elevada, sobre todo, por parte de aquellos alumnos que no existían a clase, el desarrollo de la experiencia les pareció en general muy novedosa, aunque al principio se mostraban algo reticentes y desconcertados para luego ir tomando confianza y dominar la situación, estableciéndose una interacción y un acercamiento entre ellos y con nosotras; lo cual repercutió en gran manera en el propio Servicio de Biblioteca en cuanto a consultas, búsquedas y manejo del fondo.

Con respecto a los ejercicios y las distintas actividades que tuvieron que realizar, encontramos quienes hacían lo mínimo y otros que demostraban un interés casi inusual, esforzándose, preguntando y reclamando nuestra atención.

El contenido de los temas elaborados fue evaluado, como es lógico, por la profesora, destacando que, salvo pequeñas matizaciones, se recogía lo fundamental de cada tema. En algún caso, presentaban novedades y la bibliografía utilizada era muy actual, de calidad sin olvidar a autores clásicos descubiertos por ellos mismos así como la relación con la comunidad de investigación en el mismo tema.

Se pudo comprobar que la calificación en el examen final era más alta en la parte que habían elaborado ellos que en la que habían tenido que estudiar por los apuntes cogidos en clases y la bibliografía recomendada por la profesora, al mismo tiempo los estudiantes reconocieron que les había costado menos tiempo prepararla.

En el informe final, salvo errores cometidos en la elaboración de la bibliografía, se puede decir que los resultados fueron bastante satisfactorios. Teniendo en cuenta los indicadores correspondientes a los pilares fundamentales en los que se asienta la Alfabetización Informacional, los resultados globales establecidos en tres niveles: bajo, medio y alto, son los que aparecen en la Tabla III.

\begin{tabular}{cccc}
\hline Bajo & Medio & Alto & NP \\
\hline 9 & 40 & 66 & 30 \\
\hline
\end{tabular}

Tabla III. Valoración del informe final

\section{Conclusión}

Este es el espacio reservado a la reflexión sobre la experiencia y al análisis de la propia actuación para extraer algunas conclusiones y plantear una serie de consideraciones. Esta experiencia partió con ilusión, pero con temor a que la situación se desbordase, al ser un proyecto realizado en solitario. No cabe duda que supuso un cuatrimestre de dedicación intensiva que tenía que compaginar con el resto de las tareas diarias como responsable de la biblioteca. No dudo en que pudiera cometer errores, que el programa pueda ser susceptible de mejoras 0 cambios, pero lo cierto es que resulta gratificante ver que pasados ya unos cuantos meses y que los alumnos siguen accediendo a la plataforma, que a través de ella se sigan recibiendo demandas de conexión por chat para realizar consultas, que sigan descargando materiales. Esto demuestra que el objetivo final dirigido a establecer la transversalidad se está cumpliendo. En definitiva, se aprecia que una buena parte de los estudiantes de la Escuela han adquirido la competencia de usar y gestionar la información con eficacia, resulta gratificante y estimula a avanzar en esta línea, que estoy convencida que se ajusta a los criterios de lo que podemos considerar innovador tanto en función de la enseñanza/aprendizaje como de la alfabetización informativa.

Sin embargo, no deja de ser una actuación aislada que afecta a un número reducido de alumnos. La extensión de la alfabetización informativa y de la utilización de nuevas herramientas que faciliten el aprendizaje depende de factores como: la predisposición de los bibliotecarios, la 
aceptación y colaboración del cuerpo docente y el reconocimiento institucional.

Los mejores resultados se obtienen de la formación de tipo curricular en colaboración con el profesorado por cercano, directo e interactivo.

En nuestra universidad se está trabajando en implantar una materia de libre configuración en alfabetización. Sin embargo, en mi opinión, no es la mejor solución, ya que los alumnos recurren a seleccionar este tipo de materias en función de la facilidad para superarlas.

Por lo tanto, haría las siguientes sugerencias acerca de cómo extender la alfabetización informativa a todos los colectivos universitarios, y, para que, al mismo tiempo, tenga una repercusión social en función de la construcción de la Sociedad del conocimiento.

1. Aceptación y colaboración por parte de las instituciones universitarias en la implementación de Programas de Competencias Básicas en la titulaciones como materias 0 , entre las que estaría incluida la Alfabetización Informacional. Me remito a las Normas sobre Alfabetización en Enseñanza Superior (ACRL/ALA 2000), aprobadas y adoptadas por la American Association for Higher Education y los organismos de acreditación estadounidenses.

2. El diseño de estos programas ha de estar a cargo del cuerpo de bibliotecarios/as de la universidad con la colaboración de pedagogos y especialistas en TIC.

3. La inclusión obligatoria de programas especializados en los cursos de doctorado, postgrados y master.

4. La utilización de herramientas de aprendizaje a distancia que permitan adquirir competencias sin ser necesaria la presencialidad, y que al mismo tiempo constituyan un instrumento de ayuda permanente para el trabajo autónomo y continuo.

5. Estos instrumentos, considerados como aulas abiertas formarán parte de los servicios integrados de información y formación de las bibliotecas universitarias como propone Ricardo Acebes Jiménez (2001).

6. Establecer mayor intercomunicación entre las bibliotecas universitarias mediante proyectos de colaboración y transmisión de buenas prácticas a nivel nacional e internacional.

Finalmente, considero que la extensión de la alfabetización en información, competencia fundamental para la creación de conocimiento y mejora en la calidad de vida, es una responsabilidad de la universidad para llegar a construir una sociedad con una cultura informacional competitiva.

\section{Referencias}

Acebes Jiménez, Ricardo. El Servicio de Información hacia un modelo integral. // Magan Wals, José Antonio (coord). Temas de biblioteconomía Universitaria y General. Madrid: Complutense, 2001. 397-420.

Aragón González, Ines. Formación de usuarios y alfabetización en informción". // Orera Orera, Luisa (ed.). Biblioteca Universitaria. Madrid: Síntesis, 2005. 423-443.

De la Sociedad d la Información a la Sociedad del Conocimiento y del Aprendizaje [en línea]. Alfabetizar en Informción desde la biblioteca universitaria: diseñño de herramientas para el aprendizaje virtual. Madrid: SEDIC, 2006. http//www.sedic.es/f_cursos virtuales_061tr2.htm.

Pinto Molina, Maria (coord). Portal e-coms. Tutorial hipertextual sobre alfabetización en información: habilidades para la gestión de los contenidos electrónicos [en línea] htttp://mpinto.ugr.es/e-coms (consulta: 24 de marzo 2007).

Zapirain Sagaseta, José. Docencia y cultura bibliotecaria americana. // El Profesional de la Información. 5:1 (2006) 36-42. 